\title{
Glial Development and Function in the Nervous System of Caenorhabditis elegans
}

\author{
Shai Shaham \\ Laboratory of Developmental Genetics, The Rockefeller University, New York, New York 10065 \\ Correspondence: shaham@rockefeller.edu
}

The nematode, Caenorhabditis elegans, has served as a fruitful setting for understanding conserved biological processes. The past decade has seen the rise of this model organism as an important tool for uncovering the mysteries of the glial cell, which partners with neurons to generate a functioning nervous system in all animals. C. elegans affords unparalleled single-cell resolution in vivo in examining glia-neuron interactions, and similarities between $C$. elegans and vertebrate glia suggest that lessons learned from this nematode are likely to have general implications. Here, I summarize what has been gleaned over the past decade since $C$. elegans glia research became a concerted area of focus. Studies have revealed that glia are essential elements of a functioning $C$. elegans nervous system and play key roles in its development. Importantly, glial influence on neuronal function appears to be dynamic. Key questions for the field to address in the near- and long-term have emerged, and these are discussed within.

$\mathrm{S}_{\mathrm{k} e \mathrm{~s}}^{\mathrm{in}}$ ingle-celled and invertebrate organisms are Key settings for understanding basic biology. The molecular clockworks underlying common processes, such as the cell cycle, cell death, innate immunity, RNA interference, developmental patterning, cell polarity establishment, vesicular secretion, and neuronal guidance, to name but a few, were all initially described in such model systems. Contributing to this roaring success are two properties of such systems: ease of use, and exaggeration of the phenomena under study. Caenorhabditis elegans, with its facile genetics (Brenner 1974) and small cell number (959 somatic cells in the adult hermaphrodite) (Sulston and Horvitz 1977; Sulston et al. 1983), has allowed the investigation of animal development and cell-cell interactions in vivo with un- precedented single-cell resolution. It is, therefore, an exciting choice for a model system to investigate the basic properties of glia and their interactions with neurons during nervous system development and function.

The studies described in this review reveal similarities between glia in C. elegans and other animals in morphology, development, anatomy, and function, and describe new paradigms that may be broadly conserved. Differences between glia in C. elegans and in other organisms are also apparent. For example, C. elegans axons are not myelinated (White et al. 1986), probably because axon lengths are short $(<1 \mathrm{~mm})$. One difference, however, is of tremendous experimental utility. Unlike neurons in other model systems, C. elegans neurons can survive in vivo

Editors: Ben A. Barres, Marc R. Freeman, and Beth Stevens

Additional Perspectives on Glia available at www.cshperspectives.org

Copyright (C) 2015 Cold Spring Harbor Laboratory Press; all rights reserved; doi: 10.1101/cshperspect.a020578

Cite this article as Cold Spring Harb Perspect Biol 2015;7:a020578 
without trophic support from glia (Bacaj et al. 2008; Yoshimura et al. 2008). This unique property likely stems from differences in development between nematodes and other metazoans. In Drosophila and vertebrates, for example, neurons are born in excess, and the final neuronal complement is thought to be determined by competition for target innervation (Maor-Nof and Yaron 2013). Extracellular signals, some of which appear to be glia derived (Meyer-Franke et al. 1995), communicate neuron excess and can trigger cell culling. In contrast, the number of C. elegans neurons is predetermined by lineage and is identical between all individuals of the species (Sulston and Horvitz 1977; Sulston et al. 1983). Thus, there is no apparent need for glia to influence neuronal survival, and experiments reveal that they do not (Bacaj et al. 2008; Yoshimura et al. 2008). The absence of trophic requirements provides an obvious and powerful experimental advantage. The opportunity to dissociate support functions from regulatory functions is a problem that has plagued analysis of glia-neuron interactions in other model systems. Indeed, in vertebrates and Drosophila, defining the precise in vivo roles of glia, through mutation or ablation, can prove challenging because effects on general neuronal health confound experimental outcomes.

The studies described here highlight the unique in vivo arena provided by $C$. elegans to study glial influences on the nervous system, which, together with a powerful infrastructure for gene function discovery, promise to reveal basic paradigms controlling glia-dependent processes in all animals.

\section{SPECIFICATION OF GLIA IN C. elegans}

C. elegans glia come in three flavors: 46 neuroepithelial glia ensheath neuronal receptive endings at the tips of sensory dendrites (e.g., the amphid sensory organ sheath [AMsh] and socket [AMso] glia). Four neuroepithelial glia (CEPsh glia) envelop the outer surface of the C. elegans brain-a circumferential neuropil termed the nerve ring-and also abut specific synapses within this structure. These glia also ensheath sensory neuron dendritic tips. Thus,
CEPsh glia appear to serve both ensheathing and synaptic roles defined by different domains within the same cell. Six mesodermally derived glutamate receptor (GLR) glia buttress the inner nerve ring (Fig. 1) (Oikonomou and Shaham 2011).

The zinc-finger transcription factor, LIN26 , plays a key role in the specification of neuroepithelial glia. Mutations in the lin-26 gene transform epithelial cells into neurons (Ferguson et al. 1987), and can transform glia into neurons as well (Labouesse et al. 1996). How lin-26 promotes glial fate is not well understood; however, glia-specific transcriptional enhancers have been identified in the vicinity of the lin-26 gene, suggesting specific control of lin-26 expression in these cells (Landmann et al. 2004). In Drosophila, the glial cells missing (GCM) transcription factor is expressed specifically in embryonic glia, and $\mathrm{gcm}$ mutations transform glia into neurons (Hosoya et al. 1995; Jones et al. 1995; Vincent et al. 1996), suggesting parallels with LIN-26. However, LIN-26 and GCM proteins are not obviously homologous, although both contain $\mathrm{Zn}$ finger domains.

The C. elegans gene alr-1, which encodes the C. elegans homolog of the paired class homeobox transcription factor Aristaless (Tucker et al. 2005), plays a key role in postembryonic fate maintenance of AMso glia (Melkman and Sengupta 2005; Tucker et al. 2005). In humans, mutations of the Aristaless homolog ARX cause a range of neurological defects (Stromme et al. 2002). In C. elegans, alr-1 mutants hatch with no detectable abnormalities, but progressively lose sensory functions as the animal grows. Ultrastructural studies reveal that, in older alr-1 mutants, the tight junctions between AMsh and AMso glia of the amphid cannot be detected (Fig. 1) (Tucker et al. 2005).

Although the relationship between LIN26 or ALR-1 and Drosophila or vertebrate glial specification proteins is not yet clear, studies of CEPsh glia development suggest similarities with mammalian glial differentiation. hlh-17, the $C$. elegans gene most closely related to the mammalian oligodendrocyte differentiation gene Olig2, is specifically expressed in CEPsh glia (McMiller and Johnson 2005; Yoshimura 
A
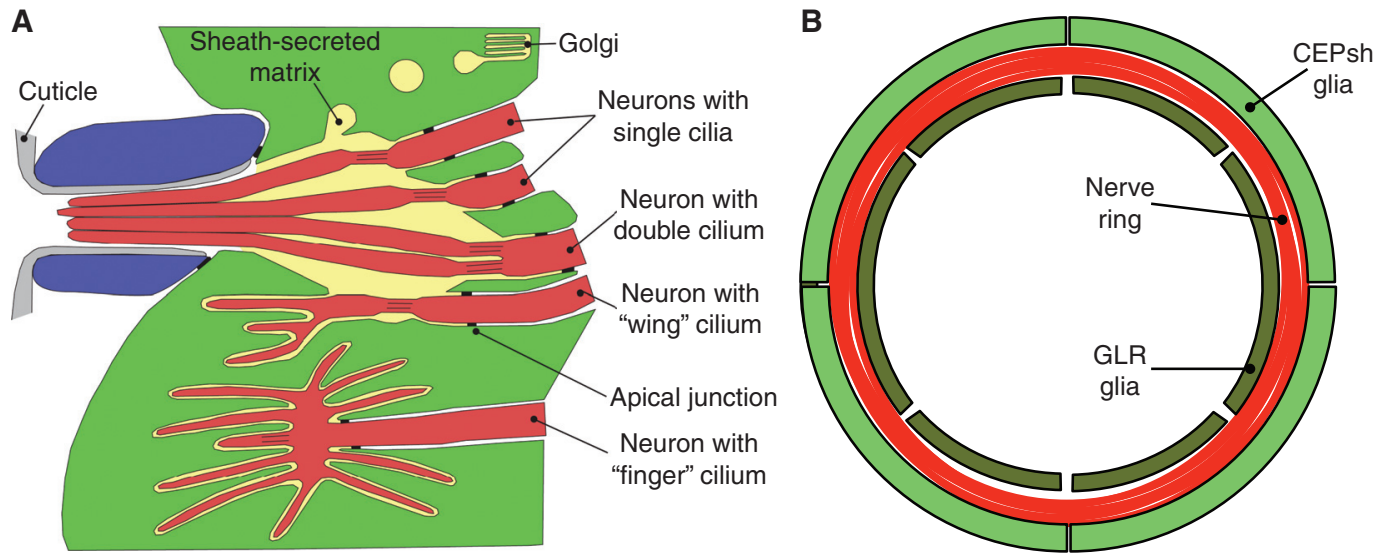

C

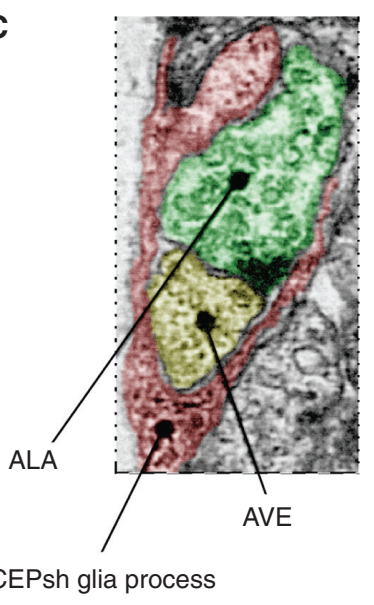

Figure 1. Three flavors of $C$. elegans glia. $(A)$ Schematic depicting the amphid sensory organ. Red, neurons; green, AMsh glial cell; and blue, AMso glial cell. Extracellular compartment formed by glia, yellow. (B) Schematic depicting the nerve ring and associated glia. Red, axons; light green, four CEPsh glia; and dark green, six glutamate receptor (GLR) glia. (C) A nerve ring synapse between the ALA (green) and AVE (yellow) neurons is ensheathed by CEPsh glia (red). (From White et al. 1986; reproduced, with permission, from The Royal Society (C) 1986.)

et al. 2008). Furthermore, this expression is under dorsoventral control reminiscent of differentiating oligodendrocytes in the developing vertebrate spinal cord. hlh-17 expression and differentiation of dorsal CEPsh glia requires the paired domain of the transcriptional regulator VAB-3 (Yoshimura et al. 2008). Expression of $h l h-17$ in ventral CEPsh glia depends on the homeodomain of VAB-3, as well as its downstream target, the MLS-2 transcriptional regulator (Yoshimura et al. 2008). VAB-3 is the $C$. elegans protein most similar to vertebrate Pax6 and Pax7 transcription factors, which regulate ventral and dorsal expression of Olig2, respec- tively, in the spinal cord (Jessell 2000; Rowitch 2004; Nicolay et al. 2007). MLS-2, an Nkx/ Hmx-related homeodomain transcription factor, is distantly related to Nkx6, which promotes Olig2 expression in the ventral pMN region of the developing spinal cord (Miller 2005). Thus, although hundreds of millions of years have passed since their last common ancestor roamed the earth, C. elegans and humans have maintained apparently similar programs for central nervous system glia specification.

Very little is known about the specification of GLR glia, which form gap junctions to muscle and sensorimotor neurons (White et al. 
S. Shaham

1986), and express a subset of the innexin gap junction proteins (Altun et al. 2009). GLR glia express the helix-loop-helix (HLH)-1 transcription factor, which is homologous to the mammalian muscle differentiation protein MyoD (Chen et al. 1994; Krause et al. 1994), suggesting a possible role for this protein in GLR differentiation as well.

\section{GLIAL ROLES IN SENSORY COMPARTMENT MORPHOGENESIS}

C. elegans senses the world primarily through sensory organs, most of which reside in the head of the animal. In these organs, glia surround sensory receptive endings at the tip of sensory neuron dendrites, forming a specialized compartment in which neuronal endings are surrounded by glial secretions (Fig. 1) (Ward et al. 1975; Perkins et al. 1986). Such compartments are common in sensory structures across animals, and are reminiscent of synapses surrounded by astrocytes (Shaham 2010). The bilateral amphids are the largest sensory organs in C. elegans and are composed of 12 neurons and two glia. Eight amphid sensory neuron receptive endings traverse the glial compartment channel, and are exposed to the outside environment (Ward et al. 1975). The receptive endings of the remaining four amphid neurons are embedded within the AMsh glia, in a hand-in-glove configuration (Ward et al. 1975). Recent studies show intricate control of glial compartment size by both glia-autonomous mechanisms and neuronal signals (Fig. 2).

DAF-6, a Patched-related protein, acts within glia to regulate compartment size (Riddle et al. 1981; Herman 1987; Perens and Shaham 2005). In daf-6 mutants, the AMsh glia compartment is bloated and malformed, and neuronal sensory endings confined within the compartment are unable to reach the outside environment. Consistent with these morphological abnormalities, daf- 6 mutants are defective in behaviors that depend on direct contact of sensory neurons with the surroundings (Albert et al. 1981; Perkins et al. 1986; Perens and Shaham 2005).
DAF-6 protein lines the glial membranes of the sensory compartment and also localizes to apical surfaces of other luminal structures in C. elegans. Animals mutant for both daf- 6 and che-14, a dispatched homolog that is also important for amphid morphogenesis (Michaux et al. 2000), display defects in many tubular systems (Perens and Shaham 2005). These results suggest that the machinery for compartment formation and morphogenesis may overlap with that controlling tube formation, raising the possibility that glial ensheathment of neurons in other animals may also be related to tubulogenesis.

DAF-6 appears to restrict glial compartment size, which suggests that a counteracting mechanism promotes compartment expansion. To identify components of such a pathway, genetic suppressors of daf-6 compartment defects were sought. One such suppressed strain harbors a mutation in the gene lit-1, encoding a NEMO/ MAP kinase homolog (Oikonomou et al. 2011). Although the glial compartment is of approximately normal dimensions in lit-1; daf-6 double mutants, lit-1 single mutants have compartments that are too narrow, failing to accommodate all amphid sensory cilia. Thus, LIT-1 likely functions to promote compartment expansion.

LIT-1 is expressed in many glial cells and localizes to subcortical regions of the glial compartment lumen (Oikonomou et al. 2011). A conserved sequence at the carboxyl terminus of the kinase is both necessary and sufficient for compartment localization. LIT-1 lacking the compartment localization domain is still able to perform other tasks in the cell, but is not able to rescue lit-1 mutant compartment defects. Localization is, therefore, essential for function (Oikonomou et al. 2011; Oikonomou and Shaham 2012). Protein interaction studies reveal that LIT-1 binds both actin and the actin regulator Wiscott-Aldrich syndrome protein (WASP). Actin is highly enriched at the surface of the glial compartment, and may represent the site of LIT-1 docking. Mutations in wsp-1, the C. elegans WASP-encoding gene, also suppress daf- 6 compartment defects, supporting the notion that LIT- 1 and WASP both function to promote compartment expansion (Oikonomou et al. 2011). 
Glia in C. elegans Nervous System

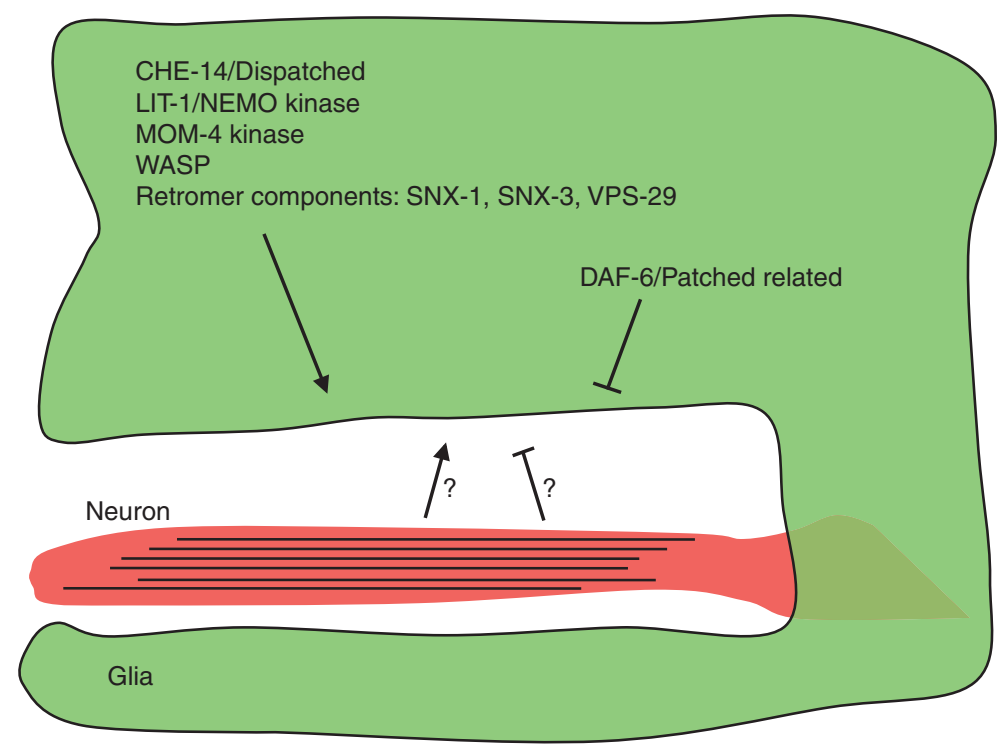

Figure 2. Regulators of glial compartment size. Neuron (red) and glia (green) are indicated. The identities of the neuronal signals are not known. WASP, Wiscott-Aldrich syndrome protein.

Although the precise roles of DAF-6, LIT-1, WASP, and actin are not fully understood, a number of studies suggest that cortical actin can serve as a platform for exocytosis (Valentijn et al. 2000; Eitzen et al. 2002; Gasman et al. 2004; Kim et al. 2007). Supporting a role for vesiclemediated secretion in controlling glial compartment size is the observation that mutations in some, but not all, components of the retromer vesicular transport complex can also suppress daf-6 defects (Oikonomou et al. 2012). SNX-1, a vesicular sorting nexin, functions in glia to antagonize DAF-6, and also localizes to the glial compartment surface (Oikonomou et al. 2012).

It appears, then, that two opposing forces, one promoting compartment expansion, the other limiting compartment growth, act in balance to form the properly proportioned glial channel of wild-type animals. How much of each pathway needs to be activated may be determined in part by neuronal signals. In animals carrying mutations that stunt or block the development of ciliated neuronal sensory endings, the glial compartment is slightly expanded and neither DAF-6 nor LIT-1 proteins line its surface, instead accumulating in a punctate structure (Perens and Shaham 2005; Oikonomou et al. 2011). The neuronal signals that communicate DAF-6 and LIT-1 localization are unknown; however, a single amino acid missense mutation in the first extracellular loop of DAF-6 nearly completely inactivates DAF-6, but has no effect on its expression or localization (Perens and Shaham 2005). This site might, therefore, define an interaction region with an extracellular, perhaps neuronal, ligand.

Cell-cell signaling may also be involved in directing the association between socket glia and sheath glia and neurons to form specific sensory organ compartments. Ablation of specific socket glia progenitors using a laser microbeam leads cognate neuron and sheath glia to associate with socket glia of a different sensory organ (Sulston et al. 1983). Thus, various forms of cell-cell communication are required to form glial compartments around sensory receptive endings.

\section{GLIAL ROLES IN SENSORY COMPARTMENT FUNCTION}

C. elegans responds to a variety of environmental stimuli including temperature, taste, smell, touch, high osmolarity, oxygen, UV light, and 


\section{S. Shaham}

pheromones (Bargmann 2006; Bounoutas and Chalfie 2007; Liu et al. 2010). Many of these stimuli are detected by sensory neurons of the amphid. This organ, therefore, provides a suitable setting for investigating glial influences on sensory neuron function. Ablation of AMsh glia in larvae, after amphid morphogenesis is complete, leads to profound functional and structural defects in ensheathed sensory neurons (Bacaj et al. 2008). Three of the four glia-embedded amphid neurons (AWA, AWC, and AFD) display involution of their sensory receptive endings, accompanied by defects in the behaviors controlled by these neurons (Fig. 3). Neuronal cell bodies, axons, and dendrites, however, remain unaffected. A similar result is elicited by transiently blocking the secretory pathway in glia (A Singhvi and S Shaham, unpubl.). The receptive endings of the AWB glia-embedded neuron are less disrupted by glia ablation, and AWB seems to maintain its function. Thus, AMsh glia are required to maintain receptive ending morphology of a subset of sensory neurons.

Neurons exposed to the outside and traversing the glial channel retain their normal morphology after glia ablation, and properly localize ciliary components and odorant receptors to their sensory receptive endings (Bacaj et al. 2008). Nonetheless, these neurons are unable to promote normal behavior. For example, calcium transients induced in one of these neurons, $\mathrm{ASH}$, in response to high osmolarity are absent in glia-ablated animals (Bacaj et al. 2008). Thus, glia seem to have separate activities controlling neuronal structure and function.

Several glial proteins have been implicated in the control of sensory neuron activity in C. elegans. The gene fig- 1 is expressed in AMsh glia and encodes a secreted protein containing
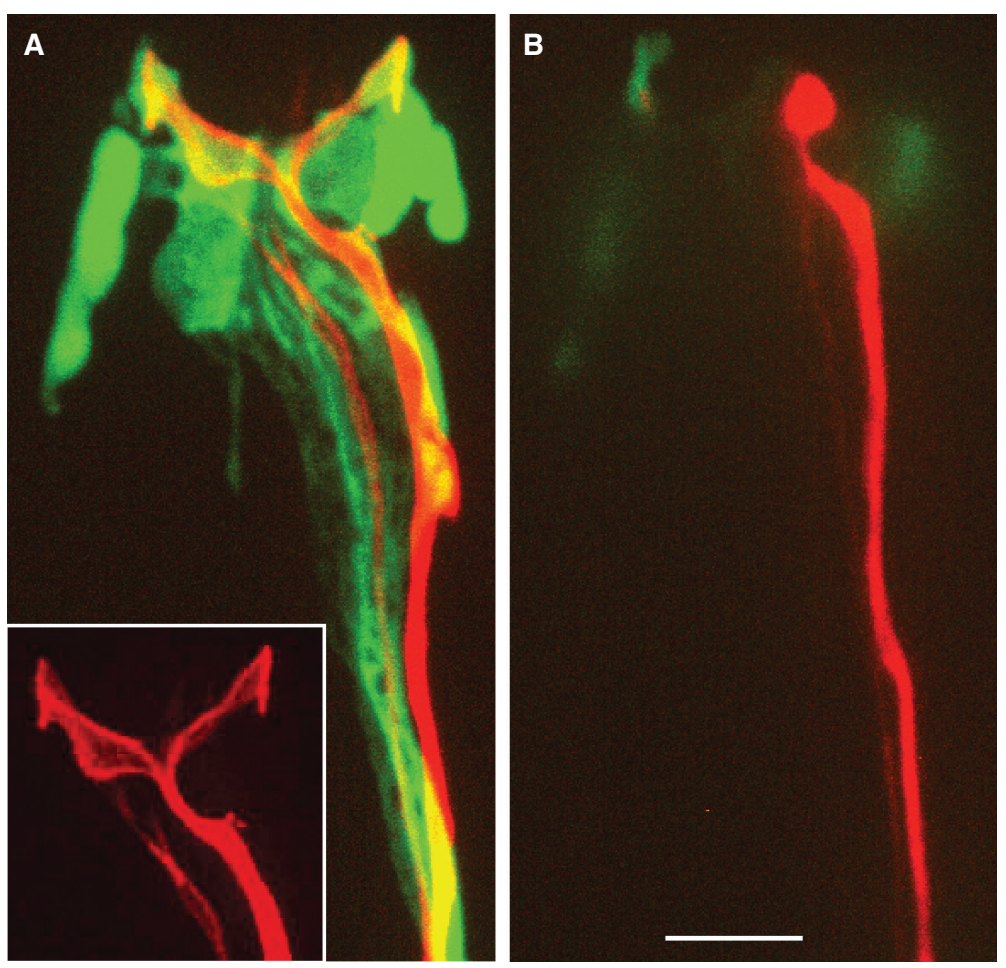

Figure 3. AMsh glia ablation leads to loss of AWC neuron sensory receptive ending. (A) Normal amphid sensory structure with AWC neuron (red) and AMsh glia (green) depicted. (Inset) AWC alone. (B) AWC neuron following glia ablation. Note loss of wing-like receptive ending. Scale bar, $0.5 \mu \mathrm{m}$. (From Bacaj et al. 2008; reproduced, with permission, from The American Association for the Advancement of Science (C) 2008.) 
two thrombospondin TSP-1 domains, multiple C6 domains of unknown function, and a putative EGF-like type II motif (Bacaj et al. 2008). Mutations in fig-1 reduce the aversive response of C. elegans to 1-octanol, a behavior mediated by neurons that traverse the amphid glial compartment. fig-1 mutants also display a defect in the ability of channel neurons to fill with the lipophilic dye DiI: wild-type animals soaked in DiI take up the dye through the amphid opening, resulting in labeling of some, but not all, channel sensory neurons (Perkins et al. 1986). Despite possessing amphids of normal morphology, sensory neurons in fig-1 mutants are unable to take up DiI. However, aqueous dyes, such as fluorescein isothiocyanate (FITC), do label amphid neurons in fig-1 mutants (J Wickelgren and S Shaham, unpubl.). The basis for this selective dye uptake defect is not understood; however, the observation raises the possibility that glial FIG-1 protein may affect aspects of neuronal membrane architecture.

Intriguing anatomical, morphological, and molecular similarities exist between sensory synapses, at which environmental signals are detected and processed by sensory neurons and neuron-neuron synapses (Shaham 2010). Sensory synapses may, therefore, serve as useful models to understand glial functions at neuron-neuron synapses. In this context, it is intriguing to note that TSP-1 and EGF-like type II domains are also found in the vertebrate protein thrombospondin, which is secreted by astrocytes and promotes synaptogenesis by acting on postsynaptic membranes (Christopherson et al. 2005; Allen 2013), raising the possibility that FIG-1 may share common functions with thrombospondins.

Glia have been postulated to control synaptic ion homeostasis, and studies of $C$. elegans sensory organs seem consistent with this idea. Osmotic stress can trigger changes in extracellular ion concentrations, and in mammalian glioma cell lines results in up-regulation of $\mathrm{H}^{+} /$ myo-inositol transporters, which have osmoprotective roles (Beck et al. 1998; Kage-Nakadai et al. 2011). These transporters also have osmoprotective roles in C. elegans, and one, hmit-1.2, is induced in AMsh glia following osmotic stress
(Kage-Nakadai et al. 2011), suggesting a possible role in controlling ion homeostasis.

C. elegans also detect and avoid low $\mathrm{pH}$, and the DEG-1 protein, a DEG/ENaC (degenerin) ion channel, functions in the ASK amphid sensory neuron in this way (Wang et al. 2008). Surprisingly, mutations in the glia-expressed degenerin ion channel ACD-1 enhance the $\mathrm{pH}$ detection defects of deg-1 mutants (Wang et al. 2008). Thus, ACD-1 may be involved in regulating extracellular ion concentrations around sensory neurons. Consistent with this hypothesis, two degenerin channels, DELM-1 and DELM-2, function in glia of a mechanosensory organ in the C. elegans head to control responses to touch (Han et al. 2013). Mutants in these channels have reduced calcium transients in glia-ensheathed mechanosensory neurons in response to light touch. Increasing extracellular potassium ion concentrations can ameliorate delm-1/2 mutant defects, suggesting a role for $\mathrm{DEG} / \mathrm{ENaC}$ channels in regulating extracellular ion balance.

$\mathrm{DEG} / \mathrm{ENaC}$ channels are also expressed in vertebrate glia (Golestaneh et al. 2000; Brockway et al. 2002; Berdiev et al. 2003; Hitomi et al. 2009) and have been implicated in synaptic plasticity (Wemmie et al. 2002; Voglis and Tavernarakis 2008), supporting the notion that glia enveloping sensory neuron receptive endings and those supporting neuronal synapses may have overlapping functions.

\section{GLIA AND SENSORY PLASTICITY}

Electron microscope (EM) reconstructions of C. elegans mutants defective in neuronal cilia formation reveal not only extensive defects in amphid sensory neuron receptive ending morphology, but also accumulation of membranebound vesicles within AMsh glia (Perkins et al. 1986). Similar observations have been made in animals expressing GFP-tagged glia-secreted proteins (Ohkura and Burglin 2011). A microarray analysis of AMsh glia identified 298 genes whose expression is glia enriched. Of these, 159 encoded proteins with signal sequences or transmembrane domains, suggesting that secretion likely plays an important role in the func- 
S. Shaham

tion of AMsh glia (Bacaj et al. 2008). Most of the secretory apparatus in these glia is located adjacent to the amphid sensory compartment (Ward et al. 1975; Perkins et al. 1986), and at least some glia-enriched proteins are secreted into the extracellular space surrounding sensory receptive endings (Perens and Shaham 2005; Ohkura and Burglin 2011). These observations suggest the interesting possibility that neurons report on their activity to glia, which respond by secreting factors that adjust neuronal activity. Although this idea has not been sufficiently tested, a number of studies suggest that glial control of neuronal plasticity is likely.

In response to environmental stress, C. elegans larvae enter the dauer developmental stage. Dauers are resistant to incubation in sodium dodecyl sulfate (SDS), and can survive up to a year without feeding. On encountering favorable conditions, animals exit the dauer state and resume development to become fertile adults. Sensory neurons of the amphid are important for both dauer entry and exit (Bargmann and Horvitz 1991). AMsh glia ablation also blocks dauer entry (Vowels and Thomas 1994). On dauer entry, the odorant receptor repertoire of amphid sensory neurons is altered (Peckol et al. 2001). In addition, some amphid neurons undergo extensive morphological remodeling (Albert and Riddle 1983; Mukhopadhyay et al. 2008), none more striking than the AWC neuron, whose glia-embedded wing-like receptive ending greatly expands in dauer animals (Albert and Riddle 1983; Procko et al. 2011). Accompanying the remodeling of the AWC neuron is a remodeling of its ensheathing AMsh glial cell. Glial invaginations housing AWC greatly expand, and AMsh glia of the bilateral amphids fuse to accommodate the massive growth of the AWC sensory ending.

Mutations in the Otx/Otd transcription factor gene $t t x-1$ prevent glia expansion and fusion, and cell-specific rescue studies show that TTX-1 protein functions within AMsh glia to control expansion (Procko et al. 2011, 2012). Remodeling of AWC neurons in ttx-1 mutants is defective, suggesting that glial expansion is required for proper AWC expansion. AMsh glia fusion requires the fusogen AFF-1 as well as the TTX-1 target gene ver- 1 , which is related to VEGF receptors. ver-1 expression is induced in AMsh glia not only in dauers, but also in nondauer animals in response to high ambient temperature, a known stimulus for dauer entry (Golden and Riddle 1984; Procko et al. 2011). The ZTF-16 Zn-finger transcription factor also functions in glia to promote ver-1 expression and glia remodeling (Procko et al. 2012). Studies of the ver-1 gene promoter suggest that $z t f-$ 16 and $t t x-1$ function through independent sites to control ver-1 expression (Procko et al. 2012). Temperature-dependent induction of ver-1 does not require sensory neuron function, suggesting that AMsh glia are able to detect temperature directly. Furthermore, glial processes expand and fuse in animals in which AWC neurons have been ablated, suggesting that glia may initiate the AWC remodeling process in dauers (Procko et al. 2011). That AMsh glia sense an environmental cue and also promote morphological changes in sensory neurons is reminiscent of the ability of vertebrate glia to detect presynaptically released neurotransmitters and to modulate dendritic spine architecture (Jahromi et al. 1992; Reist and Smith 1992; Murai et al. 2003), further supporting the argument that sensory synapses and central synapses share operating principles.

Other aspects of the dauer response may also hint at modulation of neuronal plasticity by glia. Animals that produce no serotonin are more likely to enter dauer (Sze and Ruvkun 2003), and less likely to exit dauer under favorable conditions (Moussaif and Sze 2009). Mutations in either daf- 6 or che-14 result in transcriptional up-regulation of the serotonin biosynthetic enzyme gene encoding tryptophan hydroxylase in amphid neurons (Moussaif and Sze 2009), suggesting a possible link between glia and serotonin signaling in neurons, although the physiological importance and mechanism underlying the regulation of neuronal serotonin levels by glia remain to be elucidated.

The possibility that glia are required for the integration of conflicting sensory cues in C. elegans has also been suggested. Animals normally express the odorant receptor ODR-10, which recognizes the volatile attractant diacetyl, in the 
AWA amphid neuron, which mediates attraction to stimuli (Sengupta et al. 1996). Ectopic expression of ODR-10 in AWB amphid neurons, which mediate aversive behaviors, results in a modest reduction in attraction to diacetyl (Troemel et al. 1997). Ablation of AMsh glia in the context of ODR-10 ectopic expression converts diacetyl to a strong repellant (Bacaj et al. 2008). Because C. elegans is normally exposed to a complex mixture of attractive and aversive cues, some of which are presumably sensed by AWA and AWB, it seems that glia could play an important role in integrating the overall quality of these stimuli.

Finally, sensory organ glia may also have roles in regulating organismal developmental plasticity. A recent study showed that the conserved microRNA mir-235 (similar to mammalian miR-92) promotes cell division arrest in animals that are nutritionally deprived during L1 diapause- a state reminiscent of the dauer state (Baugh 2013; Kasuga et al. 2013). mir-235 is expressed in AMso glia, and expression in these cells is sufficient to rescue mir-235 mutants.

Taken together, the studies described here provide tantalizing evidence for glial influence over neuronal plasticity. Efforts to elucidate the molecular bases of these phenomena are ongoing, and may well reveal whether and how glia control neuronal output.

\section{GLIA AND NEURITE EXTENSION}

Amphid sensory dendrites and glial processes extend over $\sim 100 \mu \mathrm{m}$ toward the tip of the nose (White et al. 1986). Extension of these neuron and glia projections proceeds through a mechanism termed retrograde extension (Sulston et al. 1983; Heiman and Shaham 2009). Amphid sensory neurons and AMsh glia are born near the tip of the developing head, in which they extend a short projection that is anchored. Posterior migration of their cell bodies results in process extension. The tip anchor is composed of at least two proteins (Heiman and Shaham 2009): the zona pellucida (ZP) domain protein DYF-7, secreted by the neurons, and DEX-1, a zonadhesin domain-containing protein secreted by nonneuronal neighboring cells. DEX-1 and DYF-7 are similar to $\alpha$ and $\beta$-tectorins, proteins comprising the tectorial membrane, an extracellular matrix that anchors hair-cell cilia in the inner ear (Legan et al. 1997). Like the tectorins and other ZP domain proteins, DYF-7 can multimerize, and its multimeric state is required for dendrite anchoring. EM of the amphid during embryonic development reveals thin extracellular filaments, which may correspond to DYF-7 and DEX-1, anchoring amphid neuron dendritic endings to an extraembryonic matrix (Oikonomou et al. 2011).

Although it is not clear whether DEX-1 is expressed in AMsh glia, other observations suggest a direct role for these glia in dendrite extension (Fig. 4). First, ablation of AMsh glia during embryogenesis results in short dendrites, similar to the ones seen in $d y f-7$ and dex-1 mutants ( $\mathrm{T}$ Bacaj and S Shaham, unpubl.). Similarly, ablation of sheath glia of the cephalic sensory organ results in dendrite extension defects of its resident sensory neurons (Yoshimura et al. 2008). Second, expression of either dex-1 or $d y f-7$ cDNA by the amphid glia can rescue the dendrite extension defect of each respective mutant (Heiman and Shaham 2009), suggesting that glia are correctly positioned to establish or modify the extracellular matrix required for dendritic tip anchoring. Third, a number of proteins predicted to contain $\mathrm{ZP}$ and related domains are expressed by AMsh glia and could, perhaps, interact with DYF-7 and DEX-1 (Bacaj et al. 2008).

The ram-5 gene also encodes a ZP domain protein and is expressed in many C. elegans glia, including the structural cell of the ray sensilla in the male tail, in which it is required for establishing sensilla morphology (Yu et al. 2000). Thus, ZP domain proteins secreted by both neurons and glia play important roles in shaping sensory organs.

C. elegans CEPsh glia play important roles in organizing and directing axon outgrowth. Laser ablation of CEPsh glia precursors has profound effects on nerve ring structure and axon guidance (Yoshimura et al. 2008). In 20\% of animals in which two of four CEPsh glial cells are eliminated, the nerve ring is not formed, 
S. Shaham
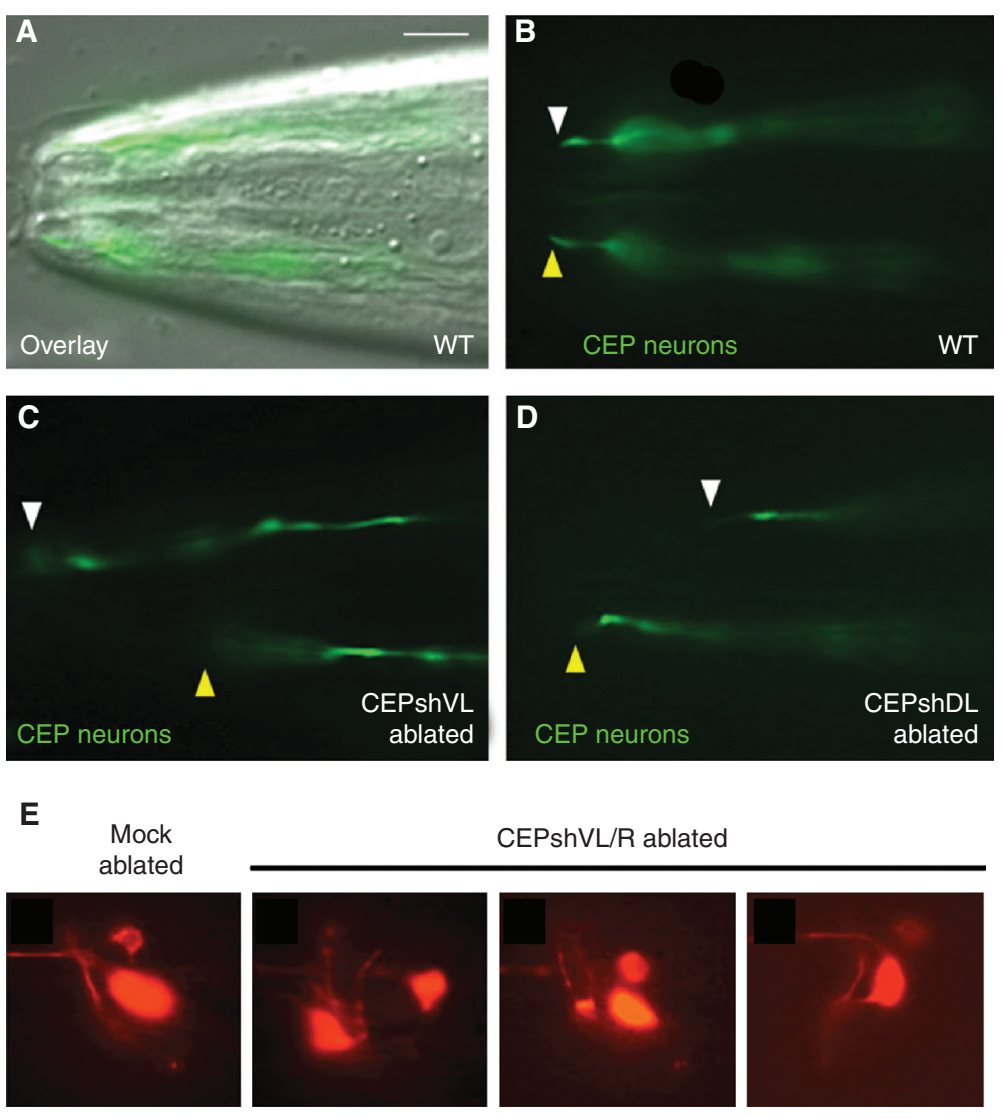

CEPshVL/R ablated
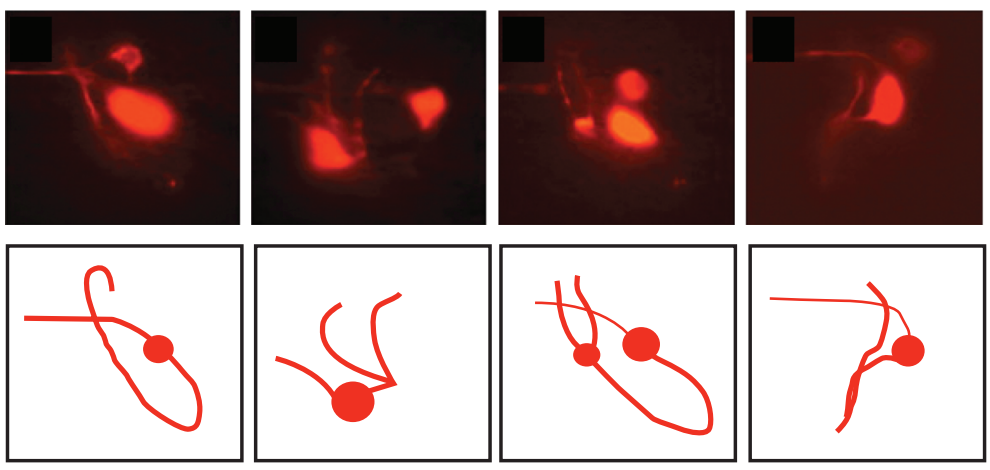

Figure 4. Glia control neurite extension and branching. $(A-D)$ Ablation of CEPsh glia leads to extension defects in ensheathed CEP dendrites. Scale bar, $5 \mu \mathrm{m}(A)$. (E) Ablation of CEPsh glia also leads to defects in nerve ring axon guidance and branching of the AWC neuron. WT, wild type. (From Yoshimura et al. (2008); reproduced, with permission, from the authors in conjunction with The Company of Biologists (C) 2008.)

and, instead, neuronal processes are splayed over the head region. In the remaining animals, a nerve ring is formed; however, axons within the ring are disorganized, manifesting guidance and branching defects (Fig. 4). At least part of the axon guidance defects is attributable to the lack of UNC-6/netrin secretion from ventral CEPsh glia (Wadsworth et al. 1996; ColonRamos et al. 2007; Yoshimura et al. 2008). However, axon guidance defects in CEPsh glia-ablated animals are much more severe than those of unc-6 mutants, suggesting that other glial effectors of axon guidance likely exist.

Glia, therefore, play integral roles in neurite guidance and extension in C. elegans, and studies of these cells in this context will likely reveal important conserved components.

\section{GLIA AND SYNAPTOGENESIS}

In vertebrates, astrocytes impact synaptogenesis by secreting proteins that establish postsynaptic 
competence (Allen 2013) and by pruning synapses (Chung et al. 2013). Studies of CEPsh glia, which envelop the synapse-rich nerve ring, suggest that these glia might participate in synapse formation in C. elegans. The establishment of synaptic contacts between the AIY and RIA neurons requires the netrin receptor UNC-40/ DCC in AIY (Colon-Ramos et al. 2007). Ventral CEPsh glia, which contact AIY, express UNC-6/ netrin (Wadsworth et al. 1996), and unc-6 mutants show synaptogenesis defects similar to unc-40, raising the possibility that CEPsh glia participate in synaptogenesis. Consistent with this idea is the observation that mutations affecting the solute carrier protein CIMA-1, which is expressed in hypodermal cells that contact CEPsh glia, can cause these glia to expand their reach. This expansion correlates with stabilization of ectopic synapses (Shao et al. 2013).

Whether glia are directing synaptogenesis or playing a more passive role, perhaps through effects on local neuronal guidance, remains to be explored. EM serial reconstruction of the AIYRIA synapse in adults suggests that the CEPsh glial process does not closely abut this synapse (White et al. 1986). Furthermore, mutations affecting CEPsh glia differentiation (Yoshimura et al. 2008) only partially prevent ectopic synapse formation in CIMA-1 mutants. Imaging the AIY-RIA synapse as it is formed in the embryo will likely help clarify how glia influence synapse establishment and/or maintenance.

Like vertebrate and Drosophila glia, C. elegans glia engulf dying cells during normal embryonic development (Sulston et al. 1983). Whether $C$. elegans glia are also able to engulf neuronal debris at synapses or following neuronal injury remains an open question.

\section{GLIA AND SYNAPTIC FUNCTION}

Most synapses in the C. elegans nerve ring are not ensheathed by CEPsh glia. However, a few synapses are reproducibly associated with glial processes, suggesting that glia may play modulatory roles at these sites (White et al. 1986). Animals in which CEPsh glia are ablated after nerve ring formation show stereotypical locomotory defects. These defects are strongly sup- pressed by ablation of the ALA neuron (M Katz and S Shaham, unpubl.). The synapse between ALA and its postsynaptic neuron AVE is one of the few synapses associated with CEPsh glia processes. Further analysis of this synapse promises to be exciting for at least two reasons. First, it will allow the identification of glial proteins affecting synaptic function. Second, it will allow the study of activity at a single synaptic site using animal behavior as a direct readout. Correlation of single synapse physiology and animal behavior remains elusive in vertebrates and Drosophila; thus, C. elegans may provide a powerful setting for testing hypotheses regarding the role of synaptic modulation on animal behavior and learning.

\section{CONCLUDING REMARKS}

C. elegans is emerging as an important model system for understanding diverse glial functions at single gene and single cell resolution. Studies of this animal have already uncovered important new principles of glia function, and are beginning to reveal their molecular bases. However, there is much more to be discovered. For example, neuronal sensory receptive endings affect glial protein localization, glial vesicle accumulation, and glial protein expression ( $\mathrm{J}$ Zuckerman, T Bacaj, C Procko, et al., unpubl.) in C. elegans, and reciprocal effects of glia on sensory receptive ending morphology and function are also observed. Together, these observations raise the hypothesis that sensory neurons transduce stimuli not only down the dendrite, but also to glia, which then adjust their secretion output, in size and/or content, to increase or decrease neuronal responsiveness. Establishing a connection, if one exists, between the signaling elements of this model in a natural behavioral setting would place glia at the center of a nervous system computation problem-a finding that could have far-reaching implications.

Although studies of $C$. elegans show an unambiguous role for CEPsh glia in axon guidance and brain assembly, how this is achieved on a cell-by-cell basis, and what the complement of necessary molecules is, remains unknown. Understanding the development of the C. elegans 


\section{S. Shaham}

nerve ring at single axon and single glial process resolution will surely reveal some principles of the assembly, and this large-scale project is currently afoot (Wu et al. 2013; P Insley, G Rapti, A Singhal, et al., unpubl.). CEPsh glia, like astrocytes, tile the nerve ring, forming well-defined quadrants ensheathing the C. elegans brain (White et al. 1986; Yoshimura et al. 2008). How glial tiling is achieved, and what the significance of this ubiquitous phenomenon is, is also currently unknown.

GLR glia are entirely unexplored. Proposed roles for these cells in nerve ring assembly need to be tested. The intriguing gap junctions between GLRs and sensorimotor neurons and muscle cells may indicate regulatory functions in coordination of head muscle activity, or controlling neuromuscular synapses, perhaps similar to roles of perisynaptic Schwann cells in vertebrates.

Finally, both GLR and CEPsh glia anatomy is suggestive of functions in nerve ring protection, together forming, perhaps, a rudimentary blood-brain barrier. Establishing the functional existence of such an isolating structure is an important priority that might further show the utility of C. elegans for dechipering conserved glial roles in the nervous system.

The first decade of $C$. elegans glia research has focused on establishing these cells as important models for understanding glial contributions to nervous system development and function. The next decade is now ripe for fundamental discovery.

\section{ACKNOWLEDGMENTS}

I thank members of the Shaham laboratory for comments on the manuscript, and apologize to those whose work was not cited either because of my oversight or to space concerns. This work is funded in part by National Institutes of Health (NIH) Grants R01NS073121 and R01NS064273 to S.S.

\section{REFERENCES}

Albert PS, Riddle DL. 1983. Developmental alterations in sensory neuroanatomy of the Caenorhabditis elegans dauer larva. J Comp Neurol 219: 461-481.
Albert PS, Brown SJ, Riddle DL. 1981. Sensory control of dauer larva formation in Caenorhabditis elegans. J Comp Neurol 198: 435-451.

Allen NJ. 2013. Role of glia in developmental synapse formation. Curr Opin Neurobiol 23: 1027-1033.

Altun ZF, Chen B, Wang ZW, Hall DH. 2009. High resolution map of Caenorhabditis elegans gap junction proteins. Dev Dyn 238: 1936-1950.

Bacaj T, Tevlin M, Lu Y, Shaham S. 2008. Glia are essential for sensory organ function in C. elegans. Science 322: 744747.

Bargmann CI. 2006. Chemosensation in C. elegans. WormBook 1-29.

Bargmann CI, Horvitz HR. 1991. Control of larval development by chemosensory neurons in Caenorhabditis elegans. Science 251: 1243-1246.

Baugh LR. 2013. To grow or not to grow: Nutritional control of development during Caenorhabditis elegans L1 arrest. Genetics 194: 539-555.

Beck FX, Burger-Kentischer A, Muller E. 1998. Cellular response to osmotic stress in the renal medulla. Pflugers Arch 436: 814-827.

Berdiev BK, Xia J, McLean LA, Markert JM, Gillespie GY, Mapstone TB, Naren AP, Jovov B, Bubien JK, Ji HL, et al. 2003. Acid-sensing ion channels in malignant gliomas. J Biol Chem 278: 15023-15034.

Bounoutas A, Chalfie M. 2007. Touch sensitivity in Caenorhabditis elegans. Pflugers Arch 454: 691-702.

Brenner S. 1974. The genetics of Caenorhabditis elegans. Genetics 77: 71-94.

Brockway LM, Zhou ZH, Bubien JK, Jovov B, Benos DJ, Keyser KT. 2002. Rabbit retinal neurons and glia express a variety of ENaC/DEG subunits. Am J Physiol Cell Physiol 283: C126-C134.

Chen L, Krause M, Sepanski M, Fire A. 1994. The Caenorhabditis elegans MYOD homologue HLH-1 is essential for proper muscle function and complete morphogenesis. Development 120: 1631-1641.

Christopherson KS, Ullian EM, Stokes CC, Mullowney CE, Hell JW, Agah A, Lawler J, Mosher DF, Bornstein P, Barres BA. 2005. Thrombospondins are astrocyte-secreted proteins that promote CNS synaptogenesis. Cell 120: 421433.

Chung WS, Clarke LE, Wang GX, Stafford BK, Sher A, Chakraborty C, Joung J, Foo LC, Thompson A, Chen C, et al. 2013. Astrocytes mediate synapse elimination through MEGF10 and MERTK pathways. Nature 504: 394-400.

Colon-Ramos DA, Margeta MA, Shen K. 2007. Glia promote local synaptogenesis through UNC-6 (netrin) signaling in C. elegans. Science 318: 103-106.

Eitzen G, Wang L, Thorngren N, Wickner W. 2002. Remodeling of organelle-bound actin is required for yeast vacuole fusion. J Cell Biol 158: 669-679.

Ferguson EL, Sternberg PW, Horvitz HR. 1987. A genetic pathway for the specification of the vulval cell lineages of Caenorhabditis elegans. Nature 326: 259-267.

Gasman S, Chasserot-Golaz S, Malacombe M, Way M, Bader MF. 2004. Regulated exocytosis in neuroendocrine cells: A role for subplasmalemmal Cdc42/N-WASP-induced actin filaments. Mol Biol Cell 15: 520-531. 
Golden JW, Riddle DL. 1984. The Caenorhabditis elegans dauer larva: Developmental effects of pheromone, food, and temperature. Dev Biol 102: 368-378.

Golestaneh N, Nicolas C, Picaud S, Ferrari P, Mirshahi M. 2000. The epithelial sodium channel $(\mathrm{ENaC})$ in rodent retina, ontogeny and molecular identity. Curr Eye Res 21: 703-709.

Han L, Wang Y, Sangaletti R, D'Urso G, Lu Y, Shaham S, Bianchi L. 2013. Two novel DEG/ENaC channel subunits expressed in glia are needed for nose-touch sensitivity in Caenorhabditis elegans. J Neurosci 33: 936-949.

Heiman MG, Shaham S. 2009. DEX-1 and DYF-7 establish sensory dendrite length by anchoring dendritic tips during cell migration. Cell 137: 344-355.

Herman RK. 1987. Mosaic analysis of two genes that affect nervous system structure in Caenorhabditis elegans. Genetics 116: $377-388$.

Hitomi Y, Suzuki A, Kawano Y, Nozawa-Inoue K, Inoue M, Maeda T. 2009. Immunohistochemical detection of $\mathrm{ENaC} \beta$ in the terminal Schwann cells associated with the periodontal Ruffini endings of the rat incisor. Biomed Res 30: 113-119.

Hosoya T, Takizawa K, Nitta K, Hotta Y. 1995. Glial cells missing: A binary switch between neuronal and glial determination in Drosophila. Cell 82: 1025-1036.

Jahromi BS, Robitaille R, Charlton MP. 1992. Transmitter release increases intracellular calcium in perisynaptic Schwann cells in situ. Neuron 8: 1069-1077.

Jessell TM. 2000. Neuronal specification in the spinal cord: Inductive signals and transcriptional codes. Nat Rev Genet 1: 20-29.

Jones BW, Fetter RD, Tear G, Goodman CS. 1995. Glial cells missing: A genetic switch that controls glial versus neuronal fate. Cell 82: 1013-1023.

Kage-Nakadai E, Uehara T, Mitani S. 2011. $\mathrm{H}^{+} /$myo-inositol transporter genes, hmit-1.1 and hmit-1.2, have roles in the osmoprotective response in Caenorhabditis elegans. Biochem Biophys Res Commun 410: 471-477.

Kasuga H, Fukuyama M, Kitazawa A, Kontani K, Katada T. 2013. The microRNA miR-235 couples blast-cell quiescence to the nutritional state. Nature 497: 503-506.

Kim S, Shilagardi K, Zhang S, Hong SN, Sens KL, Bo J, Gonzalez GA, Chen EH. 2007. A critical function for the actin cytoskeleton in targeted exocytosis of prefusion vesicles during myoblast fusion. Dev Cell 12: 571-586.

Krause M, Harrison SW, Xu SQ, Chen L, Fire A. 1994. Elements regulating cell- and stage-specific expression of the C. elegans MyoD family homolog hlh-1. Dev Biol 166: $133-148$.

Labouesse M, Hartwieg E, Horvitz HR. 1996. The Caenorhabditis elegans LIN-26 protein is required to specify and/or maintain all non-neuronal ectodermal cell fates. Development 122: 2579-2588.

Landmann F, Quintin S, Labouesse M. 2004. Multiple regulatory elements with spatially and temporally distinct activities control the expression of the epithelial differentiation gene lin-26 in C. elegans. Dev Biol 265: 478-490.

Legan PK, Rau A, Keen JN, Richardson GP. 1997. The mouse tectorins. Modular matrix proteins of the inner ear homologous to components of the sperm-egg adhesion system. J Biol Chem 272: 8791-8801.
Liu J, Ward A, Gao J, Dong Y, Nishio N, Inada H, Kang L, Yu $\mathrm{Y}, \mathrm{Ma} \mathrm{D}, \mathrm{Xu}$ T, et al. 2010. C. elegans phototransduction requires a $\mathrm{G}$ protein-dependent cGMP pathway and a taste receptor homolog. Nat Neurosci 13: 715-722.

Maor-Nof M, Yaron A. 2013. Neurite pruning and neuronal cell death: Spatial regulation of shared destruction programs. Curr Opin Neurobiol 23: 990-996.

McMiller TL, Johnson CM. 2005. Molecular characterization of HLH-17, a C. elegans bHLH protein required for normal larval development. Gene 356: 1-10.

Melkman T, Sengupta P. 2005. Regulation of chemosensory and GABAergic motor neuron development by the C. elegans Aristaless/Arx homolog alr-1. Development 132: 1935-1949.

Meyer-Franke A, Kaplan MR, Pfrieger FW, Barres BA. 1995. Characterization of the signaling interactions that promote the survival and growth of developing retinal ganglion cells in culture. Neuron 15: 805-819.

Michaux G, Gansmuller A, Hindelang C, Labouesse M. 2000. CHE-14, a protein with a sterol-sensing domain, is required for apical sorting in C. elegans ectodermal epithelial cells. Curr Biol 10: 1098-1107.

Miller RH. 2005. Dorsally derived oligodendrocytes come of age. Neuron 45: 1-3.

Moussaif M, Sze JY. 2009. Intraflagellar transport/Hedgehog-related signaling components couple sensory cilium morphology and serotonin biosynthesis in Caenorhabditis elegans. J Neurosci 29: 4065-4075.

Mukhopadhyay S, Lu Y, Shaham S, Sengupta P. 2008. Sensory signaling-dependent remodeling of olfactory cilia architecture in C. elegans. Dev Cell 14: 762-774.

Murai KK, Nguyen LN, Irie F, Yamaguchi Y, Pasquale EB. 2003. Control of hippocampal dendritic spine morphology through ephrin-A3/EphA4 signaling. Nat Neurosci 6: $153-160$.

Nicolay DJ, Doucette JR, Nazarali AJ. 2007. Transcriptional control of oligodendrogenesis. Glia 55: 1287-1299.

Ohkura K, Burglin TR. 2011. Dye-filling of the amphid sheath glia: Implications for the functional relationship between sensory neurons and glia in Caenorhabditis elegans. Biochem Biophys Res Commun 406: 188-193.

Oikonomou G, Shaham S. 2011. The glia of Caenorhabditis elegans. Glia 59: 1253-1263.

Oikonomou G, Shaham S. 2012. On the morphogenesis of glial compartments in the sensory organs of Caenorhabditis elegans. Worm 1: 51-55.

Oikonomou G, Perens EA, Lu Y, Watanabe S, Jorgensen EM, Shaham S. 2011. Opposing activities of LIT-1/NLK and DAF-6/patched-related direct sensory compartment morphogenesis in C. elegans. PLoS Biol 9: e1001121.

Oikonomou G, Perens EA, Lu Y, Shaham S. 2012. Some, but not all, retromer components promote morphogenesis of C. elegans sensory compartments. Dev Biol 362: 42-49.

Peckol EL, Troemel ER, Bargmann CI. 2001. Sensory experience and sensory activity regulate chemosensory receptor gene expression in Caenorhabditis elegans. Proc Natl Acad Sci 98: 11032-11038.

Perens EA, Shaham S. 2005. C. elegans daf-6 encodes a patched-related protein required for lumen formation. Dev Cell 8: 893-906. 
S. Shaham

Perkins LA, Hedgecock EM, Thomson JN, Culotti JG. 1986. Mutant sensory cilia in the nematode Caenorhabditis elegans. Dev Biol 117: 456-487.

Procko C, Lu Y, Shaham S. 2011. Glia delimit shape changes of sensory neuron receptive endings in C. elegans. Development 138: 1371-1381.

Procko C, Lu Y, Shaham S. 2012. Sensory organ remodeling in Caenorhabditis elegans requires the zinc-finger protein ZTF-16. Genetics 190: 1405-1415.

Reist NE, Smith SJ. 1992. Neurally evoked calcium transients in terminal Schwann cells at the neuromuscular junction. Proc Natl Acad Sci 89: 7625-7629.

Riddle DL, Swanson MM, Albert PS. 1981. Interacting genes in nematode dauer larva formation. Nature 290: 668671.

Rowitch DH. 2004. Glial specification in the vertebrate neural tube. Nat Rev Neurosci 5: 409-419.

Sengupta P, Chou JH, Bargmann CI. 1996. odr-10 encodes a seven transmembrane domain olfactory receptor required for responses to the odorant diacetyl. Cell 84: 899-909.

Shaham S. 2010. Chemosensory organs as models of neuronal synapses. Nat Rev Neurosci 11: 212-217.

Shao Z, Watanabe S, Christensen R, Jorgensen EM, ColonRamos DA. 2013. Synapse location during growth depends on glia location. Cell 154: 337-350.

Stromme P, Mangelsdorf ME, Shaw MA, Lower KM, Lewis SM, Bruyere H, Lutcherath V, Gedeon AK, Wallace RH, Scheffer IE, et al. 2002. Mutations in the human ortholog of Aristaless cause X-linked mental retardation and epilepsy. Nat Genet 30: 441-445.

Sulston JE, Horvitz HR. 1977. Post-embryonic cell lineages of the nematode, Caenorhabditis elegans. Dev Biol 56: $110-156$.

Sulston JE, Schierenberg E, White JG, Thomson JN. 1983. The embryonic cell lineage of the nematode Caenorhabditis elegans. Dev Biol 100: 64-119.

Sze JY, Ruvkun G. 2003. Activity of the Caenorhabditis elegans UNC-86 POU transcription factor modulates olfactory sensitivity. Proc Natl Acad Sci 100: 9560-9565.

Troemel ER, Kimmel BE, Bargmann CI. 1997. Reprogramming chemotaxis responses: Sensory neurons define olfactory preferences in C. elegans. Cell 91: 161-169.

Tucker M, Sieber M, Morphew M, Han M. 2005. The Caenorhabditis elegans aristaless orthologue, alr-1, is required for maintaining the functional and structural integrity of the amphid sensory organs. Mol Biol Cell 16: 4695-4704.
Valentijn JA, Valentijn K, Pastore LM, Jamieson JD. 2000. Actin coating of secretory granules during regulated exocytosis correlates with the release of rab3D. Proc Natl Acad Sci 97: 1091-1095.

Vincent S, Vonesch JL, Giangrande A. 1996. Glide directs glial fate commitment and cell fate switch between neurones and glia. Development 122: 131-139.

Voglis G, Tavernarakis N. 2008. A synaptic DEG/ENaC ion channel mediates learning in $C$. elegans by facilitating dopamine signalling. EMBO J 27: 3288-3299.

Vowels JJ, Thomas JH. 1994. Multiple chemosensory defects in daf-11 and daf-21 mutants of Caenorhabditis elegans. Genetics 138: 303-316.

Wadsworth WG, Bhatt H, Hedgecock EM. 1996. Neuroglia and pioneer neurons express UNC-6 to provide global and local netrin cues for guiding migrations in C. elegans. Neuron 16: 35-46.

Wang Y, Apicella A Jr, Lee SK, Ezcurra M, Slone RD, Goldmit M, Schafer WR, Shaham S, Driscoll M, Bianchi L. 2008. A glial DEG/ENaC channel functions with neuronal channel DEG-1 to mediate specific sensory functions in C. elegans. EMBO J 27: 2388-2399.

Ward S, Thomson N, White JG, Brenner S. 1975. Electron microscopical reconstruction of the anterior sensory anatomy of the nematode Caenorhabditis elegans. J Comp Neurol 160: 313-337.

Wemmie JA, Chen J, Askwith CC, Hruska-Hageman AM, Price MP, Nolan BC, Yoder PG, Lamani E, Hoshi T, Freeman JH Jr, et al. 2002. The acid-activated ion channel ASIC contributes to synaptic plasticity, learning, and memory. Neuron 34: 463-477.

White JG, Southgate E, Thomson JN, Brenner S. 1986. The structure of the nervous system of the nematode Caenorhabditis elegans. Philos Trans R Soc B 314: $1-340$.

Wu Y, Wawrzusin P, Senseney J, Fischer RS, Christensen R, Santella A, York AG, Winter PW, Waterman CM, Bao Z, et al. 2013. Spatially isotropic four-dimensional imaging with dual-view plane illumination microscopy. Nat Biotechnol 31: 1032-1038.

Yoshimura S, Murray JI, Lu Y, Waterston RH, Shaham S. 2008. $\mathrm{mls}-2$ and vab-3 control glia development, hlh17/Olig expression and glia-dependent neurite extension in C. elegans. Development 135: 2263-2275.

Yu RY, Nguyen CQ, Hall DH, Chow KL. 2000. Expression of ram-5 in the structural cell is required for sensory ray morphogenesis in Caenorhabditis elegans male tail. EMBO J 19: 3542-3555. 


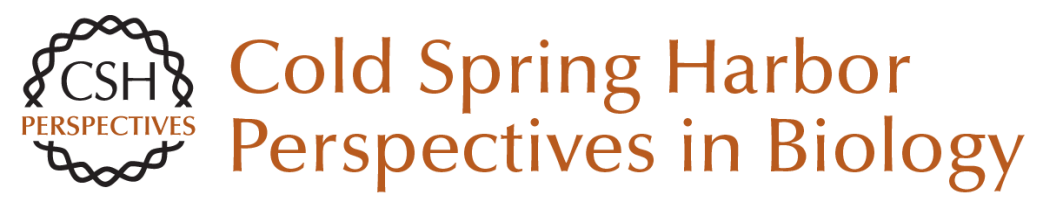

\section{Glial Development and Function in the Nervous System of Caenorhabditis elegans}

Shai Shaham

Cold Spring Harb Perspect Biol 2015; doi: 10.1101/cshperspect.a020578 originally published online January 8, 2015

\section{Subject Collection Glia}

The Nodes of Ranvier: Molecular Assembly and Maintenance Matthew N. Rasband and Elior Peles

Microglia in Health and Disease Richard M. Ransohoff and Joseph El Khoury

The Astrocyte: Powerhouse and Recycling Center Bruno Weber and L. Felipe Barros

Microglia Function in Central Nervous System Development and Plasticity Dorothy P. Schafer and Beth Stevens

Transcriptional and Epigenetic Regulation of Oligodendrocyte Development and Myelination in the Central Nervous System Ben Emery and Q. Richard Lu

Origin of Microglia: Current Concepts and Past Controversies

Florent Ginhoux and Marco Prinz

Glia Disease and Repair--Remyelination Robin J.M. Franklin and Steven A. Goldman

Astrocytes in Neurodegenerative Disease Hemali Phatnani and Tom Maniatis
Oligodendrocyte Development and Plasticity Dwight E. Bergles and William D. Richardson

Oligodendrocytes: Myelination and Axonal

Support Mikael Simons and Klaus-Armin Nave

Drosophila Central Nervous System Glia Marc R. Freeman

Perisynaptic Schwann Cells at the Neuromuscular Synapse: Adaptable, Multitasking Glial Cells Chien-Ping Ko and Richard Robitaille

Astrocytes Control Synapse Formation, Function, and Elimination Won-Suk Chung, Nicola J. Allen and Cagla Eroglu

Schwann Cell Myelination James L. Salzer

Schwann Cells: Development and Role in Nerve Repair Kristján R. Jessen, Rhona Mirsky and Alison C. Lloyd

Perineurial Glia

Sarah Kucenas

For additional articles in this collection, see http://cshperspectives.cshlp.org/cgi/collection/

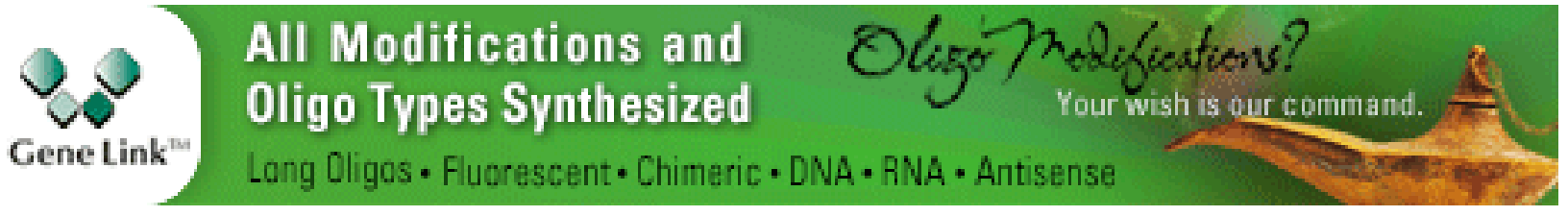

